\title{
Transport of glutamine into the xylem of sunflower (Helianthus annuus)
}

\author{
G. R. FINDENEGG ${ }^{1}$, W. PLAISIER ${ }^{1}$, M. A. POSTHUMUS ${ }^{2}$ and W. CH. MELGER ${ }^{2}$ \\ ${ }^{1}$ Department of Soil Science and Plant Nutrition, Wageningen Agricultural University, P.O. Box 8005, \\ 6700 EC Wageningen, The Netherlands, and ${ }^{2}$ Department of Organic Chemistry, Wageningen \\ Agricultural University, P.O. Box 8026, 6700 EG Wageningen, The Netherlands
}

Key words: ammonium nutrition, glutamine, Helianthus annuus L., ${ }^{15} \mathrm{~N}$, phloem-to-xylem transfer, xylem exudate

\begin{abstract}
Sunflower (Helianthus annuus L.) plants were grown on nutrient solution with ammonium nitrogen. After 12 days of growth the ammonium in the nutrient solution was labeled with ${ }^{15} \mathrm{~N}(99 \%)$. Three hours later glutamine- $\mathrm{N}$ in the xylem exudate was labeled for $56 \%$ as shown by GC-MS; this percentage increased to $63 \%$ after 8 , and to $69 \%$ after 24 hours of incubation. When the xylem exudate had been collected from the epicotyl instead of the hypocotyl, $15-\mathrm{N}$ abundances were $52 \%$, $56 \%$ and $63 \%$ respectively. Results are consistent with an import of glutamine into the transpiration stream during its ascension in the xylem. On basis of the differences in abundance of double-labeled, single-labeled and unlabeled glutamine between the two sampling sites it was estimated that at least $20 \%$ of the xylem glutamine was imported into xylem along this distance $(\sim 4 \mathrm{~cm})$.
\end{abstract}

\section{Introduction}

The occurrence of a xylem-to-phloem transfer of nitrogen compounds in plants is well established: Nitrogen taken up by plant roots is primarily transported to the shoot via the xylem transpiration stream. Growing points have a high nitrogen demand, but generally low rates of transpiration. Therefore, on the way from roots to young leaves, part of the nitrogen must interchange from xylem to phloem (Simpson, 1986). Export of nitrogen from the xylem can be studied by measuring the removing of (labeled) nitrogen compounds from the transpiration stream (Van Bel et al., 1979; Dickson et al., 1985). It has been found that amides like glutamine and asparagine are exported specifically and efficiently (Sharkey and Pate, 1975).

Much less is known about a possible import of nitrogen compounds into the xylem. However, such a transfer may affect determinations of root nitrate reduction (Rufty and Volk, 1986) and recirculation of nitrogen (Simpson et al., 1982) when such determinations are based on the composition of the xylem sap.

Rufty and Volk (1986) measured considerable percentages of endogeneous organic nitrogen (i.e. organic nitrogen derived from nitrate absorbed some time before the experiment) in the xylem sap, but argued that this could also be explained by assuming that nitrogen assimilation in the root causes a delay of its transport into the xylem. Rowland (1986) could detect organic ${ }^{15} \mathrm{~N}$ in the xylem sap after exposure of barley plants to atmospheric ${ }^{15} \mathrm{NO}_{2}$.

In this paper a substantial import of glutamine into the xylem of sunflower along the stem is indicated. ${ }^{15} \mathrm{~N}$-abundance in glutamine has been shown to decrease in the ascending xylem sap.

\section{Methods}

Seeds of sunflower (Helianthus annuus L. var. Relax) were germinated in moist quartz sand. After one week seedlings were transferred to 
nutrient solution $\left(3 \mathrm{mM}\left(\mathrm{NH}_{4}\right)_{2} \mathrm{SO}_{4}, 1 \mathrm{~m} M\right.$ $\mathrm{K}_{2} \mathrm{SO}_{4}, 1 \mathrm{~m} M \mathrm{CaCl}_{2}, 1 \mathrm{~m} M \mathrm{MgSO}_{4}, 0.25 \mathrm{~m} M$ $\mathrm{KH}_{2} \mathrm{PO}_{4}, \mathrm{Fe}-\mathrm{EDTA}$ and micronutrients).

Throughout the period of germination, growth, and ${ }^{15} \mathrm{~N}$-incubation the nutrient solution was aerated, mixed and kept at $\mathrm{pH} 6.0$ by automatic titration with diluted $\mathrm{NH}_{4} \mathrm{OH}$. The growth conditions were kept constant at $20^{\circ} \mathrm{C}$ and $80 \%$ air humidity with a photoperiod of 16 hours $\left(60 \mathrm{~W} \mathrm{~m}^{-2}\right)$.

After 12 days of growth on nutrient solution 18 plants of equal size were selected and transferred to a smaller container with $1.6 \mathrm{~L}$ nutrient solution labeled with ${ }^{15} \mathrm{NH}_{4}(99 \%)$. Xylem exudate was collected during 30 minutes from plants cut freshly $\sim 2 \mathrm{~cm}$ above or $\sim 2 \mathrm{~cm}$ below the cotyledons and immediately deep frozen.

For GC-MS analysis of the xylem exudates the procedure of MacKenzie and Tenaschuk (1985) was used. Three samples of $40 \mu \mathrm{L}$ from different plants were freeze-dried. Subsequently $4 \mu \mathrm{L}$ trimethylamine and $60 \mu \mathrm{L} \mathrm{N}$-methyl-N-(tert. butyldimethylsilyl)-trifluoroacetamide (MTBSTFA; Pierce Chemical Company) was added. The closed reaction vessel was heated to $75^{\circ} \mathrm{C}$ for $30 \mathrm{~min} .2 \mu \mathrm{L}$ were injected into the Pye Unicam 204-series gaschromatograph equipped with a CP-Sil 19CB capillary column of $0.25 \mathrm{~mm}$ i.d. $\times$
$25 \mathrm{~m}$. Temperature program was $2 \mathrm{~min}$ at $180^{\circ} \mathrm{C}$ followed by a rise to $260 \mathrm{C}$ at a rate of $4^{\circ} \mathrm{C} \min ^{-1}$. Mass spectra were obtained from a connected VG7070F mass spectrometer using $70 \mathrm{eV}$ electron impact ionization. During the chromatographic analysis of the derivatized xylem exudate the intensities of the mass spectral peaks corresponding to the (GLN(TBDMSi) $)_{3}$ $\left.\operatorname{COOSiC}_{6} \mathrm{H}_{15}\right)^{+}$-fragment (masses $329.24,330.24$ and 331.23; see also Rhodes et al., 1989) were recorded. The obtained integrated peak intensities were corrected for the natural abundances of ${ }^{2} \mathrm{H},{ }^{13} \mathrm{C},{ }^{15} \mathrm{~N},{ }^{17} \mathrm{O},{ }^{18} \mathrm{O},{ }^{29} \mathrm{Si}$ and ${ }^{30} \mathrm{Si}$.

Amino acids in the xylem sap were determined with an amino acid analyzer.

\section{Results and discussion}

\section{Abundance of glutamine and ${ }^{15} \mathrm{~N}$ in the xylem exudate}

The amino acid analysis of the xylem exudate is given in Table 1. Glutamine is by far the most abundant amino acid.

The ${ }^{15} \mathrm{~N}$-abundance in glutamine from the xylem exudate (NX) during the incubation with ${ }^{15} \mathrm{~N}$-ammonium is shown in Figure 1 . When

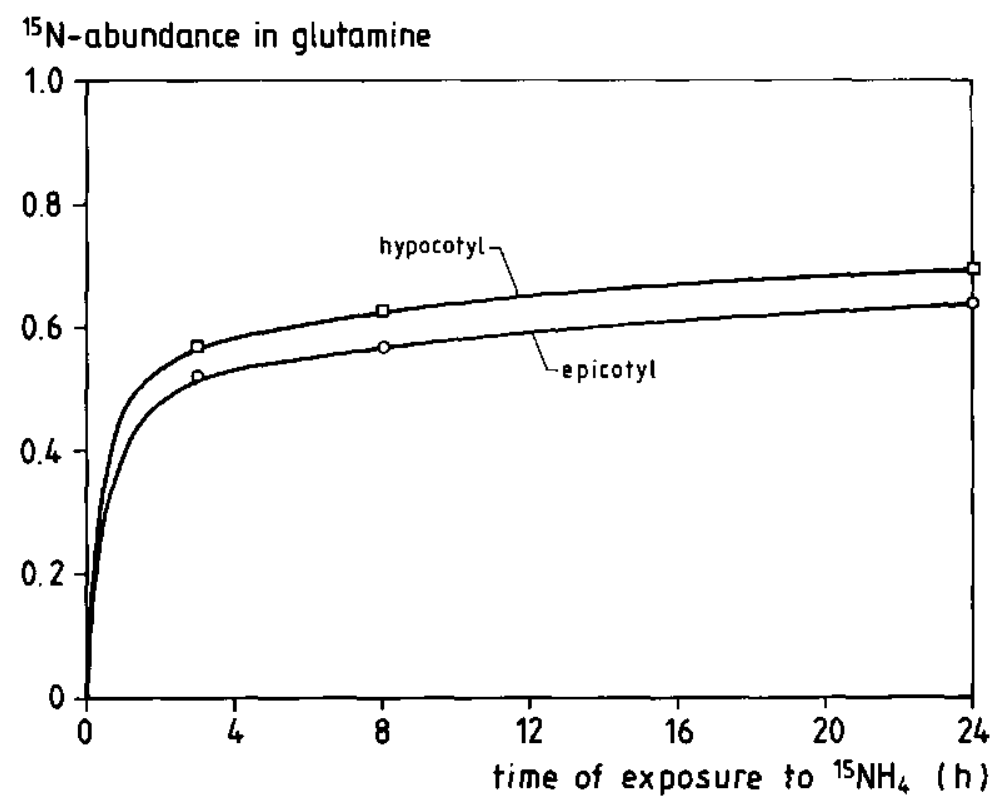

Fig. 1. ${ }^{15} \mathrm{~N}$ abundance of glutamine in the xylem exudate of sunflower plants. Xylem exudate has been collected from hypocotyl $(\square)$ or epicotyl (O). Means of 3 replicates. 
Table 1. Concentration of amino acids in the xylem exudate of sunflower. Means of two exudate samples collected from the hypocotyl

\begin{tabular}{lc}
\hline Amino acid & Concentration $(\mathrm{m} M)$ \\
\hline alanine & 1.9 \\
asparagine & 23.1 \\
aspartic acid & 0.6 \\
glutamic acid & 3.8 \\
glutamine & 149.3 \\
glycine & 0.5 \\
histidine & 1.0 \\
isoleucine & 0.8 \\
leucine & 0.9 \\
lysine & 0.5 \\
phenylalanine & 0.4 \\
serine & 6.0 \\
threonine & 2.2 \\
valine & 2.1 \\
\hline
\end{tabular}

xylem sap has been collected from the hypocotyl, $56 \%$ of the glutamine- $\mathrm{N}$ was labeled 3 hours after application of ${ }^{15} \mathrm{~N}$, i.e. nearly half of the glutamine $\mathrm{N}$ present in the xylem has been taken up by the plant more than 3 hours before. Later this percentage increased only slightly (to $63 \%$ after 8 , and to $69 \%$ after 24 hours). The initial rapid and the later slow increase in ${ }^{15} \mathrm{~N}$ abundance is in line with the results of Rufty and Volk (1986) and means that the nitrogen-pool equilibrating with xylem-glutamine is great, so that its ${ }^{15} \mathrm{~N}$ abundance does not increase quickly.

When the xylem sap has been collected from the epicotyl, consistently lower ${ }^{15} \mathrm{~N}$-abundances were recorded (Fig. 1). This indicates an import of unlabeled glutamine along the stem.

\section{Abundance of unlabeled, single labeled and double labeled glutamine}

By comparing the size of the 329,330 , and 331 peak of the mass spectrometer, abundances of unlabeled, single and double labeled glutamine molecules (X0, X1 and $\mathrm{X} 2$ ) could be calculated (Table 2). It was found that at any given ${ }^{15} \mathrm{~N}$ abundance in the xylem (NX) the percentage of single labeled glutamine was less than expected on basis of a stochastic distribution of label over the amino and amido group of glutamine. With a stochastic distribution the abundance of double
Table 2. Abundance of unlabeled (X0), single labeled (X1) and double labeled $(\mathrm{X} 2)$ glutamine in the xylem exudate of sunflower. Means of 3 replicates

\begin{tabular}{lcclll}
\hline $\begin{array}{l}\text { Collection } \\
\text { site }\end{array}$ & $\begin{array}{l}\text { Time of exposure } \\
\text { to }{ }^{15} \mathrm{NH}_{4} \text { (hours) }\end{array}$ & & $\mathrm{X} 0$ & $\mathrm{X} 1$ & $\mathrm{X} 2$ \\
\cline { 1 - 1 } Hypocotyl & 3 & & 0.208 & 0.456 & 0.336 \\
& 8 & & 0.170 & 0.404 & 0.427 \\
& 24 & 0.120 & 0.371 & 0.509 \\
Epicotyl & 3 & 0.252 & 0.454 & 0.294 \\
& 8 & 0.218 & 0.441 & 0.344 \\
& 24 & 0.168 & 0.390 & 0.442 \\
\hline
\end{tabular}

labeled glutamine $\mathrm{X} 2$ should be $\mathrm{NX}^{2}$; however the experimentally derived value as $\mathrm{NX}^{1.87 \pm 0.04}$ (Fig. 2). This is consistent with the results of Rhodes et al., (1989). Apparently glutamine molecules have been synthesized at sites of different ${ }^{15} \mathrm{~N}$-abundance. Glutamine synthesized at sites with high ${ }^{15} \mathrm{~N}$-abundance has an increased chance to get labeled at both nitrogen atoms whereas glutamine synthesized at sites with low abundances had an increased chance to stay totally unlabeled. Long-distance transport of intermediate products (e.g. glutamate) during glutamine synthesis thus seems to be of minor importance.

\section{Analysis of variance}

An analysis of variance has been conducted for the ${ }^{15} \mathrm{~N}$-abundance data. Significance levels of the effects of the factor 'xylem sap collection site' and of the covariate 'time of incubation' are shown in Table 3. NX, X0, X1 and X2 were significantly influenced by the time of incubation whereas the decline of ${ }^{15} \mathrm{~N}$-abundance between hypo- and epicotyl was significant only with respect to the increase of unlabeled glutamine.

The fact that the exponent EX, linking NX to $\mathrm{X} 2$, did not increase significantly during the experiment or between the collection sites means that there was no indication of a later metabolization of glutamine after its initial production. Such a metabolization should randomize the preferential formation of double labeled glutamine and consequently increase EX towards a value of 2. Sharkey and Pate (1975) reported that 
abundance of glutamine species

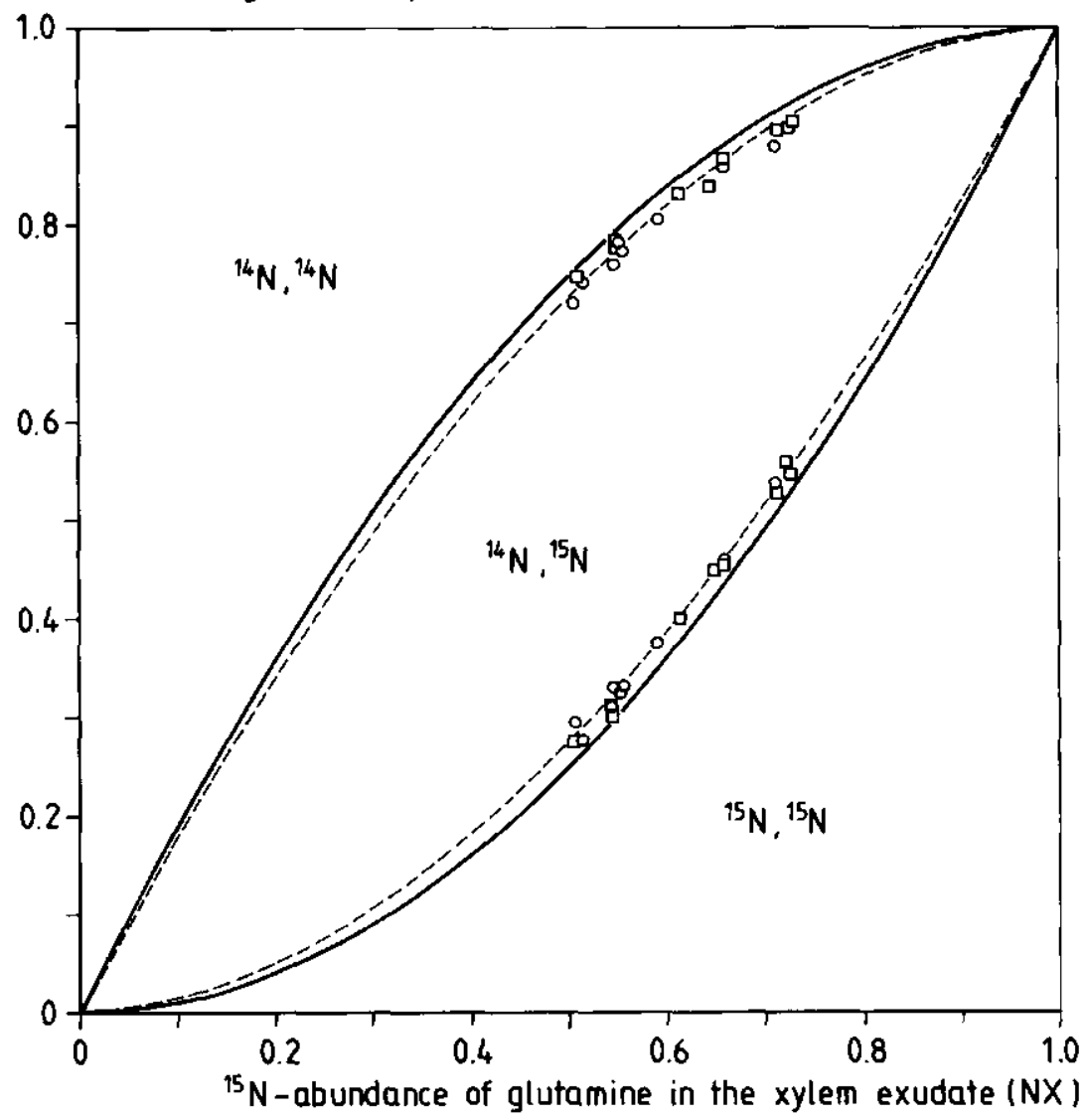

Fig. 2. Abundance of unlabeled (X0), single labeled (X1) and double labeled (X2) glutamine in the xylem exudate of sunflower plants as a function of the ${ }^{15} \mathrm{~N}$-abundance of glutamine (NX). Xylem exudate has been collected from the hypocotyl ( $\square$ ) or epicotyl $(\mathrm{O})$. Solid lines represent stochastic distribution of ${ }^{15} \mathrm{~N}$ between amino and amido group, broken lines are drawn according to the formula $\mathrm{X} 2=\mathrm{NX}^{1.87}$.

Table 3. Analysis of variance of data on the abundance of unlabeled (X0), single labeled (X1) and double labeled (X2) glutamine in the xylem exudate, the ${ }^{15} \mathrm{~N}$-abundance of glutamine in the xylem exudate (NX) and the exponent linking $\mathrm{X} 2$ to $\mathrm{NX},(\mathrm{EX})$. Significance levels: $* *<0.01$; $*<0.05 ; \mathrm{NS}>0.05$

\begin{tabular}{lll}
\hline & Effect of & \\
\cline { 2 - 3 } Analysis for & Covariate: time & $\begin{array}{l}\text { Factor: site of } \\
\text { collection }\end{array}$ \\
\hline $\mathrm{X} 0$ & $* *$ & $*$ \\
$\mathrm{X} 1$ & $* *$ & NS \\
$\mathrm{X} 2$ & $* *$ & NS \\
NX & & \\
EX & $* *$ & NS \\
\hline
\end{tabular}

glutamine exported from the xylem was largely unmetabolized when recovered in the phloem sap.

Quantitative estimation of glutamine import into the xylem

From the labeling pattern of the glutamine molecules at the two collection sites the percentage of glutamine imported between hypocotyl and epicotyl can be estimated. From Table 2 it can be calculated that after 3 hours of incubation X0 at the hypocotyl was $17 \%$ lower than at the epicotyl. Because a possible export of glutamine 
from the xylem should not affect the ratios between $X 0, X 1$ and $X 2$, it can be concluded that $17 \%$ of the glutamine present in the epicotyl must have been replaced by unlabeled glutamine between the collection sites. Data after 8 and 24 hours incubation gave a differences in X0 of 22 and $29 \%$, respectively. Glutamine in the exchange pool may have got slightly labeled during the experiment, so that the import rate obtained in this way may be an underestimate. Therefore it is reasonable to assume that at least $20 \%$ of the glutamine found in the xylem sap of the epicotyl has been imported along the $\sim 4 \mathrm{~cm}$ distance between the collection sites.

It should be noted that the import rate obtained in this way is about double the rate which would have been calculated by simply comparing NX of the two collection sites (7.7 to $10.1 \%$ ). This discrepancy is due to the fact that differences of $\mathrm{X} 0$ between the sampling sites are more pronounced than those of NX.

It is unlikely that the import of glutamine into the xylem described here can be explained by a xylem-to-xylem transfer as postulated by Layzell et al., (1981). In our experiment external ${ }^{15} \mathrm{~N}$ crossed the root into the xylem within 3 hours. Regarding this high rate of transport one would not expect that glutamine transfer from one xylem vessel to another is so slow that the imported glutamine is still largely unlabeled after 24 hours. Export of glutamine from the cotyledons via phloem to the xylem of the stem is much more likely. According to our measurements such a transfer may be much more intense than indicated by nitrogen balance studies (Layzell et al., 1981).

\section{References}

Dickson R E, Vogelman Th C and Larson P R 1985 Glutamine transfer from xylem to phloem and translocation to developing leaves of Populus deltoides. Plant Physiol. 77, 412-417.

Layzell D B, Pate J S, Atkins C A and Canvin D T 1981 Partitioning of carbon and nitrogen and the nutrition of root and shoot apex in a nodulated legume. Plant Physiol. 67, 30-36.

MacKenzie S L and Tenaschuk D 1985 Gas liquid chromatographic assay for asparagine and glutamine. $J$. Chromatogr. 322, 228-235.

Rhodes D, Rich P J and Brunk D G 1989 Amino acid metabolism of Lemna minor L. IV. ${ }^{15} \mathrm{~N}$-labeling kinetics of the amide and the amino groups of glutamine and asparagine. Plant Physiol. 89, 1161-1171.

Rowland A J 1986 Nitrogen uptake, assimilation and transport in barley in the presence of atmospheric nitrogen dioxide. In Fundamental, Ecological and Agricultural Aspects of Nitrogen Metabolism in Higher Plants. Eds. $\mathrm{H}$ Lambers, J J Neeteson and I Stulen. pp 211-214. Martinus Nijhoff Publishers, Dordrecht, The Netherlands.

Rufty T W and Volk R J 1986 Alterations in enrichment of $\mathrm{NO}_{3}^{-}$and reduced- $\mathrm{N}$ in xylem exudate during and after extended plant exposure to ${ }^{15} \mathrm{NO}_{3}$. Plant and Soil 91, 329-332.

Sharkey P J and Pate J S 1975 Selectivity in xylem to phloem transfer of amino acids in fruiting shoots of white lupine (Lupinus albus L.). Planta 127, 251-262.

Simpson R J, Lambers H and Dalling M J 1982 Translocation of nitrogen in a vegetative wheat plant (Triticum aestivum). Physiol. Plant. 56, 11-17.

Simpson R J 1986 Translocation and metabolism of nitrogen: Whole plant aspects. In Fundamental, Ecological and Agricultural Aspects of Nitrogen Metabolism in Higher Plants. Eds. H Lambers, J J Neeteson and I Stulen. pp 71-96. Martinus Nijhoff Publishers, Dordrecht, The Netherlands.

Van Bel A J E, Mostert E and Borstlap A C 1979 Kinetics of $\mathrm{L}$-alanine escape from the xylem vessels. Plant Physiol. 63, 244-247. 